\title{
ÉXITO, (HIPER)VISIBILIDAD, AMBIVALENCIA: LAS INSTALACIONES DE MONA HATOUM ${ }^{1}$
}

\section{RESUMEN}

A finales de los años ochenta Mona Hatoum decidió abandonar la performance y el vídeo para hacer instalaciones. Este artículo analiza las condiciones en que se produjo este cambio examinando el contexto en el que las instalaciones adquirieron éxito y visibilidad. Hatoum realizó estas obras en un momento en el que el arte político parecía haber entrado en crisis, al menos en la escena artística londinense. Debido a su estética minimalista, las instalaciones jugaron un papel ambivalente pues, dependiendo de dónde se exhibiesen, el compromiso político que pretendían materializar quedaba fácilmente desactivado. El artículo propone que esta ambivalencia está inextricablemente vinculada a las estrategias desarrolladas por Hatoum para encontrar su lugar en un sistema del arte especialmente preocupado por institucionalizar la diferencia cultural.

Palabras clave: Mona Hatoum, minimalismo, institucionalización, globalización, young British art

\begin{abstract}
At the end of the 1980s Mona Hatoum decided to give up performance and video and turn instead to installations. This article assesses the conditions in which this change came about and examines the context in which Hatoum's installations became successful and visible. These works were created at a time when political art was immersed in a crisis in the London art scene. Due to their minimalist aesthetic, Hatoum's installations played an ambivalent role in this context, as the political commitment they sought to embody could easily be deactivated by the place in which where they were exhibited. The article argues that this ambivalence is inextricably linked to the strategies developed by Hatoum in order to negotiate her place in an art system especially concerned with institutionalising cultural differences.
\end{abstract}

Keywords: Mona Hatoum, minimalism, institutionalisation, globalisation, young British art

Como ha observado recientemente la historiadora del arte taiwanesa Chin-Tao Wu, la conciencia que tenemos hoy del impacto de la globalización en el mundo del arte ha creado nuevos problemas a la hora de interpretar la obra de artistas que provienen de lugares ajenos a la cultura occidental ${ }^{2}$. El proceso acelerado de visibilización en el que se han visto inmersos estos artistas desde comienzos de los años noventa, debido sobre todo al triunfo de las grandes exposiciones a escala internacional -esos macroeventos que conocemos como bienales-, ha trastocado por completo nuestra manera de entender el arte contemporáneo. De hecho, el cambio con respecto a la representación de los artistas del llamado Tercer Mundo ha sido tan drástico, que como indica Rasheed Araeen, hoy ya no es posible "organizar un acto o una exposición internacional sin la participación de artistas de todo el mundo"3. Y esto supone, tal como ha subrayado a su vez Jean Fisher, que "la marginalidad cultural ya no es un problema de visibilidad, sino de exceso de visibilidad en los términos de una lectura de la diferencia cultural que es demasiado fácilmente comercializable" ${ }^{4}$. Para algunos autores esta hipervisibilidad ${ }^{5}$ del arte contemporáneo proveniente de todas partes del mundo no es tanto el resultado de una 
verdadera reflexión sobre la complejidad de las nuevas prácticas artísticas, como el efecto de una nueva "conciencia post-colonial", que no deja de ser en muchos casos profundamente problemática ${ }^{6}$.

Observando que un número considerable de artistas mujeres nacidas en países con graves problemas sociopolíticos ha alcanzado una exitosa carrera en este contexto internacional, Chin-Tao Wu se ha preguntado por los motivos de este éxito: "cómo se llega ahí y qué destrezas se necesitan para mantenerse" ${ }^{\prime 7}$. Este artículo también se enfrenta a esta pregunta, y lo hace a través del caso concreto de la artista palestino-británica Mona Hatoum. Mi intención, no obstante, es abordar el tema de una manera diferente a la planteada por Wu, quien también se ha interesado por la obra de esta artista. Wu trata de explicar el éxito de Hatoum en el contexto del arte actual. No obstante, su verdadero interés se encuentra en otro lugar: en los problemas de recepción del arte contemporáneo en un mundo culturalmente globalizado. Su argumento principal es que este tipo de obras políticas realizadas por mujeres opone cierta resistencia a la homogeneización cultural que supuestamente asociamos a la globalización. Wu insiste en que la "especificidad y las dificultades inherentes [a las obras realizadas por estas artistas] no necesariamente viajan bien", que no siempre pueden ser exportadas satisfactoriamente, mucho menos traducidas ${ }^{8}$. A diferencia de lo ocurrido con MacDonald's o la música pop, estas obras de arte, de carácter claramente político, no se prestan a un proceso de transferencia tan directo porque el cúmulo dispar de espectadores que asisten a verlas en lugares tan variados como La Habana, São Paulo, Gwangju, Montreal o Estambul no necesariamente están familiarizados con los referentes culturales desde los que las obras fueron creadas. De modo que este arte político nos plantea otro tipo de interrogantes a la hora de explicar la globalización cultural de la que también él forma parte. Así pues, el asunto que de verdad inquieta a Wu no es tanto el proceso (político, mediático, institucional) por el que este tipo de obras se ha convertido sorprendentemente en la hegemonía, sino un tema mucho más amplio, y quizás complejo, como es el de la traducción cultural.
Sin perder de vista el problema de la recepción, lo que me propongo a lo largo de estas páginas es abordar la cuestión del éxito desde un punto de vista ligeramente distinto: literalmente, desde las "destrezas que se necesitan para mantenerse", es decir, desde las estrategias desarrolladas por la artista y los contrasentidos que se producen una vez que sus obras consiguen deslizarse desde los márgenes hacia una posición de centralidad. Creo que éste es un asunto fundamental para comprender el lugar que ocupan las instalaciones de Hatoum en el llamado "mundo global del arte" tas obras son ciertamente ejemplares de los procesos de institucionalización de la diferencia cultural y de la hipervisibilidad, como explica magistralmente Chin-Tao Wu, pero también lo son de las negociaciones a las que deben llegar los artistas de la diáspora cuando su trabajo es asimilado por el sistema. Con frecuencia, la bibliografía sobre Hatoum ha dejado esta cuestión de lado. Los críticos y los historiadores del arte suelen tomar las piezas de esta artista como ejemplo del arte comprometido, de la situación del exiliado o de las dinámicas de la globalización, olvidando las condiciones específicas de creación y difusión en las que adquirieron la visibilidad y el éxito que tienen hoy. Mi propósito es revisar brevemente ese contexto y entender a través de él el cambio que se produjo en la obra de Hatoum cuando decidió empezar a realizar instalaciones. Con ello espero poner de relieve la ambivalencia inherente a estas obras, la dificultad que encuentra su sentido político para hacerse verdaderamente aprehensible; en definitiva, la complejidad que encierran las instalaciones cuando son miradas desde la realidad concreta en que consiguieron salir a la luz para hacerse (hiper)visibles.

\section{De la performance $y$ el vídeo a la instalación}

La obra de Mona Hatoum (Beirut, 1952) dio un giro radical a finales de los años ochenta cuando la artista presentó en la galería londinense Showroom su primera instalación, The Light at the End (1989) (Fig. 1). Hasta ese momento, es decir, a lo largo de los años ochenta, Hatoum había realizado performances y vídeos de claro contenido político, que hacían visible su 


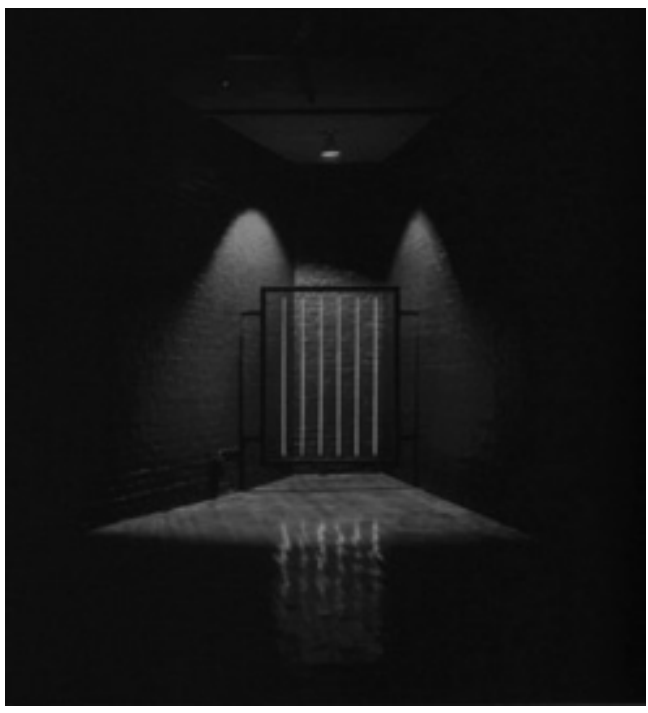

Fig. 1. Mona Hatoum, The Light at the End, Collection Arts Council of Great Britain, 1989. En: Hal Foster, Rosalind Krauss, Yve-Alain Bois, Benjamin H.D. Buchloh, Art Since 1900. Modernism, Antimodernism, Postmodernism, New York, Thames \& Hudson, 2004, p. 636

propio compromiso con las políticas de identidad y con la crítica a las estructuras de poder ${ }^{10}$. Su objetivo era azotar la conciencia del espectador, invitándolo a reflexionar sobre los códigos de disciplinamiento social. Es el caso de So Much I Want to Say (Quiero decir tantas cosas) (1983) (Fig. 2), un vídeo donde Hatoum se mostraba a sí misma tratando de decir algo mientras unas manos masculinas tapan su boca impidiéndole hablar. Al mismo tiempo que la imagen muestra esa lucha de Hatoum por hacerse oír sin conseguirlo, una voz en off -la propia voz de la artista - repite incansablemente el título de la obra. El vídeo ilustra a través de una metáfora visual muy clara la situación del subalterno - o más concretamente, la subalterna-, a quien una fuerza externa y sin rostro -esas manos masculinas que bien podrían simbolizar la institución patriarcal- priva de voz $^{11}$.

Con The Light at the End, Hatoum abandonó el modelo narrativo de articulación de la obra, basado en hacer visible un mensaje políti-

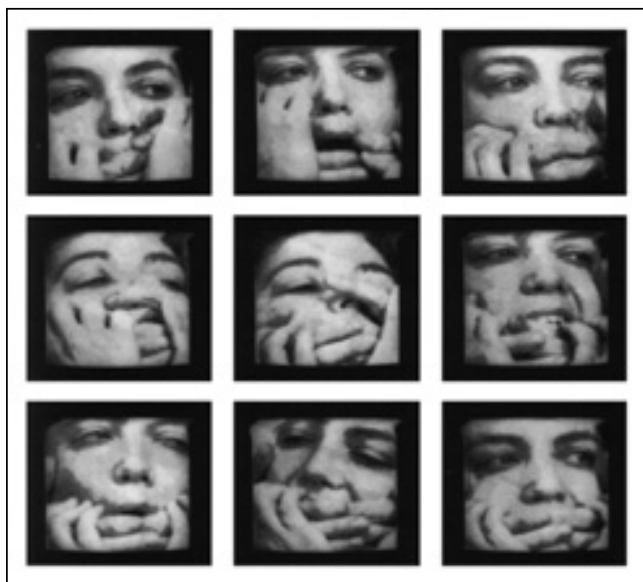

Fig. 2. Mona Hatoum, So Much I Want to Say, A Western Front Video Production, Vancouver, 1983. En: Heinrich Christoph (ed.), Mona Hatoum, cat. exp., Ostfildern-Ruit, Hatje Cantz Verlag, 2004, p. 1.

co a una audiencia presente - una audiencia que mira pero no interviene- y se sumió en un modelo completamente distinto de abordar el encuentro con el espectador. En las instalaciones, el espectador deja de ser testigo para pasar a experimentar físicamente las obras. Jugando con el aspecto atractivo de las formas y los materiales, que siguen rigurosamente una estética minimalista, Hatoum crea objetos y espacios que resultan ser lo contrario de lo que aparentan. En The Light at the End, el espectador penetra a través de un túnel, al final del cual se topa con unas líneas rojas de luz que recuerdan a las conocidas estructuras fluorescentes de Dan Flavin. Sin embargo, lo que se presentaba en el título como esa luz que nos alivia al final del túnel resulta ser un conjunto de barras al rojo vivo, una reja carcelaria que limita el paso y nos encierra, y que de tocarla o intentar traspasarla nos abrasaría la piel. Detonantes de un peligro potencialmente mortal, las instalaciones de Hatoum provocan inquietud y desasosiego en el 
espectador porque lo confrontan con la presencia insoslayable de la obra, obligándolo a proyectarse psicológicamente en ella, haciéndole experimentar mental, y casi físicamente, el dolor y la angustia que el contacto real con ella provocaría.

A lo largo de los años noventa Hatoum creó numerosos objetos o espacios que repetían con variaciones el mismo efecto conseguido en The Light at the End; esa sensación de pulcritud y belleza estética, y de sorpresa, miedo y repulsión. A veces se trata de transformar objetos cotidianos, ya sea en su materialidad, en escala o en estructura. Así ocurre, por ejemplo, en Doormat, un felpudo hecho de clavos afilados que nos invita con la palabra "Welcome" a que entremos en la galería caminando por encima de él (Fig. 3); o en Incomunicado, una cuna cuyo colchón desaparece, dejando a la vista una maya de hilos cortantes (Fig. 4). Recuperando el gusto surrealista por la subversión de lo cotidiano, Hatoum consigue que estos objetos se vuelvan extraños, peligrosos y sobre todo alienantes cuando imaginamos su uso potencial. Otras veces, sin embargo, la artista produce este mismo efecto de una manera completamente distinta: crea escenarios (Fig. 5) que distorsionan nuestra percepción a través del uso de luces y sombras, y de estructuras metálicas que nos introducen en un espacio confuso y agobiante, pero al mismo tiempo atractivo, a medio camino entre lo carcelario, lo burocrático y lo institucional.

Este cambio formal y conceptual en la manera de trabajar y de exponer sus obras - desde lo semiótico a lo fenomenológico, desde la representación a la presencia' ${ }^{12}$ - ha sido siempre subrayado pero pocas veces explicado por los historiadores y los críticos. En general, suele recurrirse a las propias explicaciones de Hatoum, que ha insistido en que tal cambio se debió a su creciente desconfianza ante los resultados que estaba consiguiendo con las performances: por un lado, la transmisión de un mensaje político que no nace de la obra sino que es ilustrado por ésta, sin llegar a involucrar al espectador, y por otro, la facilidad con la que este tipo de obras explícitamente políticas, y muchas veces además explícitamente relativas al tema palestino o a su historia personal, estaban siendo engullidas por el sistema, permitiendo iden-

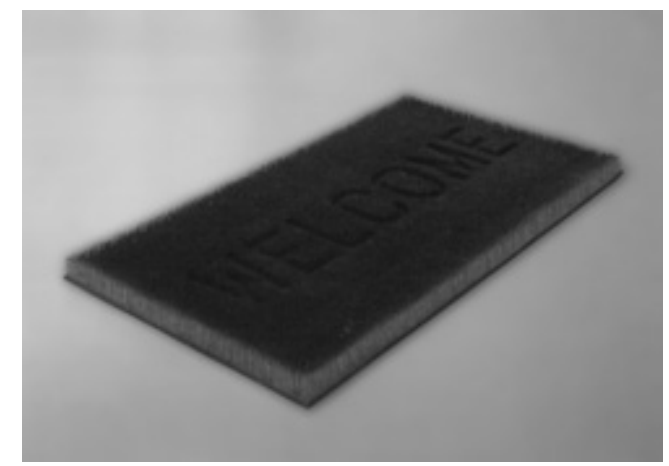

Fig. 3. Mona Hatoum, Doormat, 1996. En: Michael Archer, Guy Brett, Catherine de Zegher, Mona Hatoum, London, Phaidon, 2003, p. 14

tificar a Hatoum bajo la etiqueta de artista "mujer", "no-occidental" y "política"13. Todo este conjunto de nuevas categorías taxonómicas, que responden, según la propia Hatoum, a la concepción "ordenada" de la otredad que se busca en Occidente ${ }^{14}$, permitían encasillarla bajo estereotipos fácilmente explotables, creando a su vez expectativas sobre sus obras futuras, de las que se esperaba exhibiesen claramente la identidad conflictiva de la artista —al menos si querían entrar en una institución que se congratulaba de su apertura hacia lo políticamente correcto.

Si efectivamente este cambio de rumbo nos habla de la conciencia que tenía Hatoum sobre las mecanismos institucionales que pretenden reconocer la otredad, pero que en el fondo se apropian de su discurso alternativo para desactivar las diferencias, al mismo tiempo es obvio que no explica el hecho — subrayado por Chin-Tao $\mathrm{Wu}^{15}$ - de que mientras las performances y los 


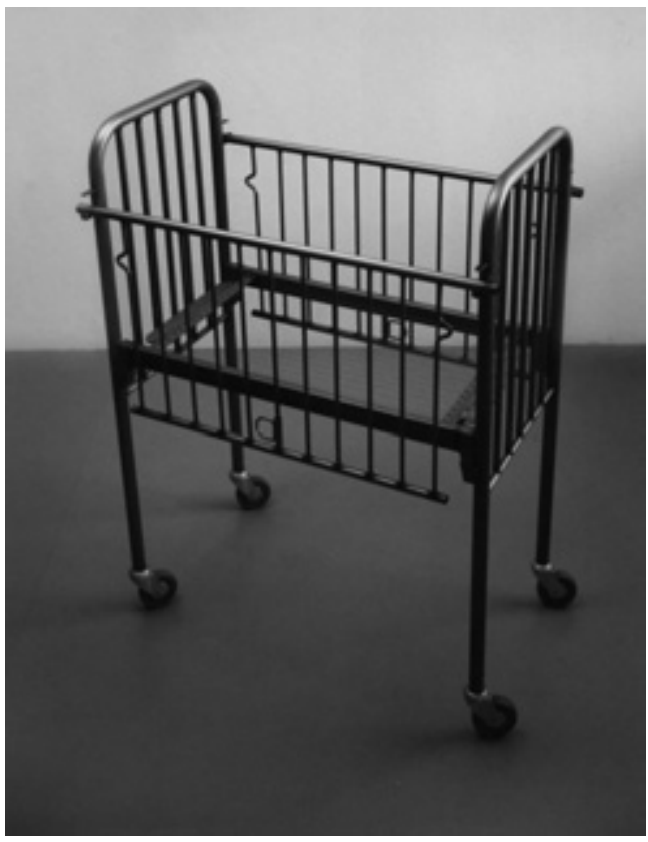

Fig. 4. Mona Hatoum, Incomunicado, 1993. En: Heinrich Christoph (ed.), Mona Hatoum, cat. exp., Ostfildern-Ruit, Hatje Cantz Verlag, 2004, p. 6.

vídeos, con sus temas explícitamente políticos, no consiguieron penetrar en el mercado ni en el museo, sus instalaciones sí lo hicieron y de forma relativamente rápida. Es decir, el reconocimiento de este cambio no consigue explicar el éxito de las instalaciones de Hatoum, que parecían estar reinventando la manera de hacer arte político y que representaban un desafío a la ideología normalizadora de la institución occidental.

\section{Hatoum en contexto: el diálogo con el young British art}

Como indica $\mathrm{Wu}$, la presencia internacional de Hatoum se debe, "en términos generales", a las políticas sobre la identidad y la diferencia cultural que caracterizaron esos años noventa, y a la apropiación de éstas por parte de las instituciones ${ }^{16}$. En términos generales, el éxito de Hatoum se debe a lo que Kobena Mercer ha llamado la "normalización multicultural"17. Pero esto sólo explica el asunto en términos genera-

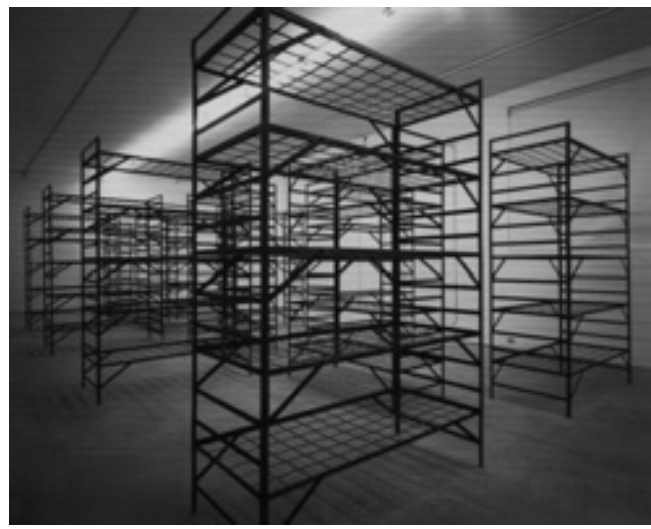

Fig. 5. Mona Hatoum, Quarters, Instalación en Viafarini, Milan, 1996. En: Michael Archer, Guy Brett, Catherine de Zegher, Mona Hatoum, London, Phaidon, 2003, p. 61.

les. Además viene a ser una explicación que si se acepta llanamente resulta conflictiva porque con ella la posición subversiva de Hatoum se disuelve, pues aparentemente fracasa: su éxito vendría a ser en el fondo el éxito de las instituciones y del mercado global, y eso no es fácil de aceptar para los críticos, que recurrentemente presentan a Hatoum como ejemplo de la artista postcolonial, poseedora de una capacidad crítica envidiable y de una posición política incorruptible $^{18}$. Tal vez por eso no ha sido frecuente que se mencione esta cuestión en la bibliografía sobre Hatoum, y de ahí la importancia de los planteamientos de Chin-Tao Wu. Pero, tal vez por eso también, Wu deja a un lado su pregunta inicial sobre el éxito para concentrarse en criticar los vagos modelos interpretativos que ha fomentado el mundo de las bienales - y mucha de la historiografía sobre Hatoum-, donde pese a todos los intentos de la artista por no circunscribir el significado de la obra, por dejarlo 
abierto y siempre a expensas de la experiencia personal del espectador, se sigue recurriendo al material biográfico, como si el origen de la artista, y por extensión los conflictos políticos y sociales del pueblo palestino, pudieran leerse directamente en la obra "por virtud — dice Wude estar de alguna manera inscritos en las obras mismas"19.

Ya a finales de los años noventa Jessica Morgan había advertido de los peligros de convertir el origen en la única vía de análisis de la obra de Hatoum. Su encasillamiento como "artista del tercer mundo" ha contribuido, insiste Morgan, a simplificar la ambivalencia y la ironía con la que Hatoum aborda el asunto de la identidad cultural, ocultando al mismo tiempo el hecho de que su formación tuvo lugar en Inglaterra, dentro de una escena cultural politizada donde el feminismo, junto con los asuntos de clase y raza, tuvieron un papel muy importante en la activación de su conciencia política, antes incluso de que empezara a reflexionar en sus obras sobre su condición de exiliada o sobre la causa palestina. "Aquí —dice Morgan- obtuvo su lenguaje y su perspectiva teóricos, que simplemente no habrían estado disponibles para ella si hubiese permanecido 'genuinamente' ( $y$, podría decirse, limitadamente) 'nativa'" 20 .

Si bien este asunto resulta verdaderamente imprescindible para comprender las estrategias críticas, irónicas y desestabilizadoras de Hatoum, no nos sirve, sin embargo, para explicar el éxito de las instalaciones. Para comprender las implicaciones del cambio que se inicia con The Light at the End no basta con reconocer el compromiso de la artista con el activismo británico de sus años de formación, también es necesario tener en cuenta las condiciones que rodearon su obra en los años noventa, y la lucha de Hatoum por sobrevivir en estas circunstancias. Para empezar, se nos plantea una cuestión interesante cuando descubrimos que a finales de los años ochenta el arte político en la línea del que por entonces estaba haciendo Hatoum empezó a dejar de interesar en la escena artística británica, donde la crisis económica había cerrado el grifo de las subvenciones públicas, y las entidades y coleccionistas privados empezaban a mirar hacia otro tipo de arte: el que estaba re- alizando la joven generación de artistas británicos que hoy conocemos como young British artists $^{21}$. En 1989, el mismo año en que Hatoum presentó The Light at The End en la galería Showroom, el artista británico-pakistaní Rasheed Araeen, ya por entonces director de la conocida revista Third Text, comisarió la primera gran exposición sobre artistas no-occidentales en Gran Bretaña, en la que Hatoum también participó. La exposición, titulada The Other Story (La otra historia), pasó desapercibida para los historiadores del arte y recibió grandes críticas en las reseñas de los periódicos porque, como explicaría años después el propio Araeen, se consideró que las obras no tenían calidad ${ }^{22}$. El fracaso parcial de The Other Story, hoy convertida en un hito de la historia del arte postcolonialista ${ }^{23}$, nos habla de la compleja situación que vivía en aquel momento el arte político realizado en los márgenes del sistema. Pensemos que se trata del momento preciso en que Hatoum está cambiando su forma de trabajar.

Aunque pocas veces se ha tenido en cuenta el hecho de que el éxito y la visibilidad de Hatoum coincidieron exactamente con el éxito rotundo de los young British artists, ésta es quizás una cuestión a repensar. Como muchos autores han puesto de manifiesto, la actitud burlona y desenfadada del young British art tuvo que ver en buena medida con un rechazo al arte políticamente correcto que había caracterizado a la generación anterior, con un desinterés llamativo por las políticas de representación (deudoras de la teoría postestructuralista) que habían animado el trabajo de artistas como la joven Hatoum ${ }^{24}$. Carl Freedman, uno de los principales responsables del patrocinio curatorial de los young British artists, resumió con lucidez la actitud del momento hacia el arte político:

En Gran Bretaña la izquierda ha reclamado tradicionalmente el arte como propio, queriendo decir con ello que el arte debería estar al servicio de la gente, y que los artistas [deberían ser] inequívocamente anti-establishment. Sin embargo, enfrentados a semejante actitud agotada y programática, resultaba realmente más radical aceptar el establishment y trabajar junto a él. ${ }^{25}$ 


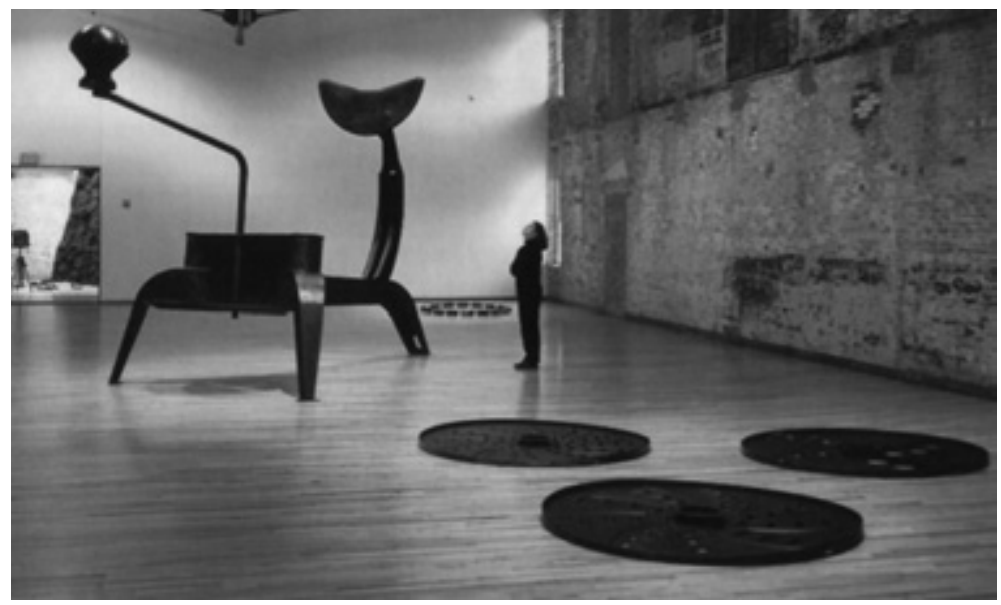

Fig. 6. Mona Hatoum, La Grande Broyeuse (Mouli-Julienne x 17), MASS MoCA, Massachusetts, 1999. En: Mona Hatoum. Interior Landscape, Milan y New York, Edizioni Charta / Charta Books, 2009, p. 74.

Aunque este desinterés por el arte político se iría contrarrestando a medida que avanzaban los noventa, según crecía la tolerancia liberal del sistema y su capacidad para acoger todo lo que significase corrección política o arte en los márgenes, lo cierto es que en el momento en que Hatoum abandonó los vídeos y las performances, estos no tenían apenas posibilidad de entrar en el sistema y hacerse visibles. Resulta curioso que Hatoum decidiese entonces optar por la instalación y el minimalismo, que venían a ser, respectivamente, el medio y el lenguaje menos anti-establisment que se podían imaginar. Y resulta también curioso que instalación y estética minimalista fuesen dos de los recursos que caracterizaron buena parte del trabajo de los young British artists ${ }^{26}$.

No es casual el hecho de que ese gran manual para el arte contemporáneo que es Arte desde 1900 dedique a Hatoum el mismo capítulo que a Rachel Whiteread, considerada comúnmente como una de los young British artists, y que el punto de unión entre las dos artistas sea el minimalismo, que resurgió con fuerza en la Gran Bretaña de los noventa. El capítulo comienza relacionando a Hatoum con un grupo de mujeres en el que no sólo está Whiteread, sino también otras artistas vinculadas al nuevo arte británico como Sarah Lucas o Gillian Wearing. El término "young British art" no se menciona en ningún momento, lo cual tiene sentido porque ni Hatoum, ni Cornelia Parker, que aparece citada en las mismas líneas, pertenecieron al grupo. Pero es significativo que se subraye el vínculo conceptual y formal entre Hatoum y estas artistas y, sobre todo, que sea éste el lugar que los autores conceden a Hatoum en la historia del arte contemporáneo: no dentro de los capítulos dedicados al arte político, el feminismo o la diferencia cultural, sino en uno concreto dedicado al grupo de mujeres que hizo brillar de forma especial al arte británico de los noventa gracias, en buena medida, a su recuperación del postminimalismo, su regreso al trabajo de artistas como Eva Hesse, Ree Morton, o Jacki Winsor ${ }^{27}$.

Para introducirnos con algo más de detalle en la problemática que quiero tratar, quizás resulte útil que nos detengamos por un momento en una obra de Damien Hirst titulada Party Time (1995), un cenicero aumentado considerablemente en tamaño, que comparte la misma estética limpia y cuidada que La Grande Broyeuse (Mouli Julienne x 17) (1999) de Hatoum, también un objeto cotidiano, en este caso una trituradora, de tamaño igualmente descomunal (Fig. 6). Pese a la cercanía formal y estética, la 
instalación de Hatoum no es equiparable, mucho menos equivalente, a la de Damien Hirst o al trabajo en general de los compañeros de éste en el Goldsmith College. El propio Julian Stallabrass, que escribió el primer gran estudio crítico sobre el young British art, puso de manifiesto que lo que caracterizaba a este nuevo arte británico era más el temperamento y la táctica que el estilo y el medio ${ }^{28}$. Y precisamente en lo que se refiere a temperamento y táctica existen importantes diferencias con respecto a la obra de Hatoum.

Mientras que el cenicero de Hirst, que transforma una conocida obra de Robert Morris en un contenedor de colillas, explota con ironía las posibilidades comerciales del minimalismo, La Grande Broyeuse de Hatoum, que transforma un inofensivo utensilio de cocina en una especie de máquina gigante de tortura, subvierte el lenguaje minimalista para poner de manifiesto su retórica del poder ${ }^{29}$. Aquí la agresividad de las formas limpias y del objeto que se nos impone ya no está asociada al poder masculino, sino al femenino, que obviamente está representado por el utensilio de cocina. Pero antes que remitir al espacio doméstico femenino como un lugar acogedor, símbolo del calor hogareño y la crianza, la trituradora aumentada diecisiete veces en tamaño parece convertirse en la mantis religiosa que nos devora y despedaza, una representación de lo femenino que casi viene a entroncar con la idea de vagina dentata ${ }^{30}$. Aquí lo cotidiano y lo doméstico se vuelven siniestros y enajenantes; la belleza de formas y materiales se torna ofensiva e implacable.

La estrategia no es exclusiva de Hatoum en estos años. Si su reapropiación subversiva del lenguaje minimalista nada tiene que ver en términos de "temperamento y táctica" con el uso que hace de él Damien Hirst, no podemos decir que sea algo completamente ajeno a lo que hacían algunos de los young British artists, pues precisamente lo siniestro [unheimlich], entendido en sentido freudiano como "el retorno de las cosas familiares que se han vuelto extrañas por la represión", es un concepto que se ha empleado para definir las instalaciones de Rachel Whiteread $^{31}$ (Fig. 7). Lo que se pone de manifiesto en la comparación con Party Time de Hirst es que este tipo de adaptación libre del lengua- je formal del minimalismo se había vuelto algo común en el momento, al menos dentro del contexto británico. Como indicaría a mediados de la década Lynn Zelevansky, el éxito del minimalismo estribaba en la versatilidad de sus recursos formales para acoger muy diferentes significados ${ }^{32}$, de modo que era posible, como hacían Hatoum o Whiteread, utilizarlo a la manera de Eva Hesse, para insuflarle una nueva vida no exenta de contenidos políticos y de efectos psicológicos, pero también era posible hacerlo a la manera de Hirst, convirtiéndolo en parte integral de las estrategias comerciales y mediáticas del propio artista. Hace falta, pues, comprender cuál es la función que se le da. Como veíamos a través de La Grande Broyeuse, lo que consigue Hatoum con su manejo particular de la retórica minimalista es minar desde dentro del sistema los recursos del lenguaje dominante al hacer convivir en sus obras la apariencia estética del arte despolitizado del momento - como el de Hirst- con los contenidos de las políticas de identidad. Y recordemos que los años noventa, especialmente a través de los escritos de Dave Hickey, trajeron de vuelta la defensa del concepto de belleza como retorno a un arte despolitizado $^{33}$. Es frente a este discurso estético, que insinúa incompatibles belleza y política, y que quiere extirpar la ideología de una vez por todas del arte, como si esto fuese posible, frente al que Hatoum parece querer lanzar sus instalaciones: "cada aspecto de la vida -le decía a Michael Archer en una entrevista- está lleno de contradicciones: algunas personas piensan que la estética y la política no pueden mezclarse pero eso es ridículo" 34 .

\section{Ambivalencia y contradicción}

Lo interesante de la comparación entre la obra de Hatoum y la de Hirst, de señalar su cercanía formal y estética, no es sólo que nos muestra la versatilidad que adquirió el lenguaje minimalista durante los años noventa. También nos indica que Hatoum probablemente tuvo que negociar su entrada en el sistema, pues si bien hubo de buscar nuevas estrategias para seguir haciendo arte político, estas estrategias significaron, en parte, adoptar ciertos recursos que estaban alcanzando gran éxito, un éxito que no era fácil conseguir en la escena artística londi- 


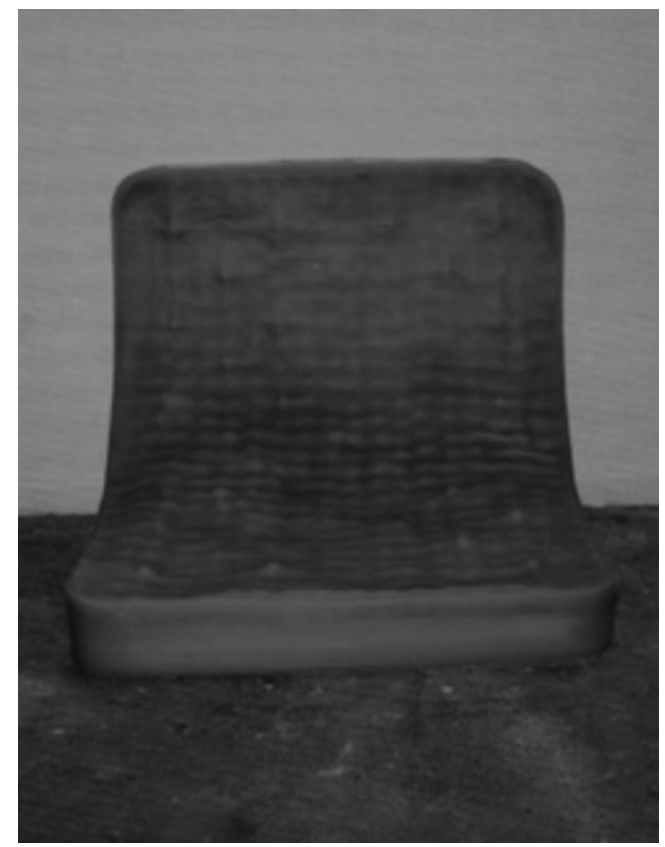

Fig. 7. Rachel Whiteread, Untitled (Amber Double Bed), 1991 En: Hal Foster, Rosalind Krauss, Yve-Alain Bois, Benjamin H.D. Buchloh, Art Since 1900. Modernism, Antimodernism, Postmodernism, New York, Thames \& Hudson, 2004, p. 637.

nense azotada por la crisis y la falta de dinero público.

Después de un tiempo en que Hatoum lo tuvo difícil para conseguir nuevos encargos ("durante unos años después de hacer The Light at the End nadie quiso mostrar nada" ${ }^{35}$ ) el rumbo que tomó el mundo artístico de los noventa permitió que las instalaciones de Hatoum comenzaran a abrirse camino, integrándose definitivamente en los circuitos del arte. De un lado, en las bienales (La Habana, Venecia, Estambul, El Cairo o São Paulo) y en otras exposiciones en Europa y Norteamérica, donde generalmente se subrayaba el sentido político y de otredad de las obras. De otro, en las galerías londinenses interesadas en el arte británico contemporáneo (Arnolfini, Serpentine, Hayward Gallery, South London Gallery o la conocida White Cube, entre otras), espacios por los que empezaban a circular paralelamente las obras de los young British artists. No obstante, las circunstancias en las que Hatoum debía seguir trabajando en los noventa habían cambiado respecto a la década anterior: la diferencia cultural, que había caracterizado y que todavía caracterizaba su trabajo, empezaba a estar, como apunta Kobena Mercer, "más visiblemente integrada que nunca en los mercados del mainstream, pero [iba] acompañada de un ethos privatizado, debido al cual [había dejado de ser] un 'asunto' para el debate público". El arte político no sólo estaba depreciado, también se había vuelto aséptico. De modo que quizás debemos plantearnos, como hace Mercer con los artistas británicos negros, la siguiente pregunta: “ ¿cómo han influido estos cambios en las elecciones que hacen los artistas de la diáspora?" ${ }^{36}$. ¿Qué estrategias se vio obligada Hatoum a desarrollar en estas condiciones?

Volviendo de nuevo a las obras que hemos comparado y a la idea de que Hatoum subvierte el lenguaje minimalista, tal vez sea posible (siguiendo un tanto heterodoxamente los planteamientos de Hyla L. Robicsek) entender sus instalaciones según el concepto de "mímica colonial" de Homi Bhabha: como aquel Otro cuya diferencia se basa en que "es casi lo mismo, pero no exactamente" ${ }^{37}$. Aquí, es precisamente ese "casi lo mismo, pero no exactamente" lo que da a las instalaciones de Hatoum su potencia y su capacidad subversiva. Podríamos considerarlo una táctica de la propia Hatoum para que su entrada en el sistema no suponga una mera adhesión, sino, por el contrario, un nuevo posicionamiento crítico; para que aun utilizando esa lingua franca que fue el minimalismo, y ese medio hegemónico que fue la instalación, sus obras sigan teniendo la misma capacidad de azotar las conciencias ${ }^{38}$.

Aunque no debemos llevar esta idea de la mímica más allá de lo aconsejable, porque no todas las obras de Hatoum guardan un parecido con las del young British art como el de los ejemplos que acabamos de ver, lo cierto es que esa idea de "lo que es casi lo mismo, pero no exactamente", nos plantea una cuestión compleja que apenas se ha tratado en los escritos sobre Hatoum. Y es que cuando algo es casi lo mismo, puede ocurrir que la diferencia no sea apreciada. Las obras de Hatoum se sitúan en una posición muy complicada porque la estrategia 
que parecen materializar —aparentar una cosa que realmente no son, y tratar así de desestabilizar aquello que aparentan- puede fácilmente darse la vuelta y volverse contra sí misma.

Durante los años noventa las instalaciones de Hatoum aparecieron simultáneamente tanto en exposiciones dedicadas al arte político ${ }^{39}$, el minimalismo hecho por mujeres ${ }^{40}$, o el arte de los países árabes ${ }^{41}$, como en bienales o en exposiciones dedicadas al young British art. La obra de Hatoum pudo verse, de hecho, en Sensation $^{42}$, quizás la más importante, la más polémica y conocida de las exposiciones sobre los young British artists. Podemos por tanto adscribir a la obra de Hatoum un papel ambivalente dentro de las políticas de exhibición de aquellos años, pues participaron -es decir, adquirieron visibilidad- en contextos en los que la posición subversiva de la artista parecía quedar desactivada. Aquí, tanto la difusión como la recepción juegan un papel muy importante que la artista pocas veces puede controlar. Como ha mostrado Chin-Tao Wu, la especificidad de obras como las de Hatoum, especialmente su sentido político, puede no viajar bien de un contexto a otro, perdiéndose así por el camino ${ }^{43}$. Pero podría añadirse aún algo más: la estrategia de Hatoum en las instalaciones, que es producir obras lo suficientemente abiertas como para que el significado no esté determinado de antemano, contribuye en parte a esta posible pérdida de sentido, porque no hay motivo para ver en ellas un contenido político, excepto que efectivamente ya lo sepamos ${ }^{44}$.

El éxito de estas obras se vuelve así un asunto delicado y nos hace plantearnos la misma pregunta que se hizo lan McLean hace unos años: "las practicas postcoloniales pueden estar en sintonía con la globalización económica, pero ¿la afirman o la resisten?" ${ }^{45}$. Se trata de uno de los principales problemas con los que se han encontrado los teóricos poscolonialistas -el problema, como ha apuntado Gerardo Mosquera, de que "las prácticas postcoloniales reproducen las estructuras del capitalismo global, aun tratando de ser contestatarias" ${ }^{46}$ - El éxito de Hatoum es inseparable del éxito de las bienales y del éxito de los young British artists, incluso si la artista ha tratado de ser crítica con ellos. Porque la belleza de sus formas minimalistas, querámoslo o no, es tan subversiva como fácil de comercializar. El problema para la crítica es que aceptar esto parece minar la posición de Hatoum, que efectivamente es una persona con plena conciencia de los problemas que ha traído la globalización para los artistas no-occidentales, especialmente para los que provienen de lugares con una historia conflictiva, como es su caso. Pero aquí el problema fundamental es que en la historiografía sobre Hatoum ha ocurrido como en buena parte de los estudios culturales a la hora de enfrentarse al legado colonial. Lawrence Grossberg ha descrito este problema de manera especialmente lúcida:

Mucho del trabajo en estudios culturales se ha preocupado de investigar y desafiar la construcción de las identidades subalternas, marginadas o dominadas, aunque algunos trabajos recientes han empezado a explorar las identidades dominantes como construcciones sociales. Raramente, sin embargo, son estudiadas las dos juntas - como la teoría parecería dictar - como mutuamente constitutivas ${ }^{47}$.

Creo que con Hatoum ha ocurrido algo parecido: con alguna excepción, como la de Chin-Tao Wu o la de Jessica Morgan, los estudios sobre ella se concentran en mostrar la dimensión crítica de un tipo de obra que sólo una artista no occidental, obligada al exilio, puede crear. Apenas se ha problematizado esta obra, indagando en su carácter ambivalente y en sus contradicciones; en su apoyo al sistema tanto como en su oposición a él; en el hecho de que apoyo y oposición tal vez no sean aquí antagónicos, sino, como dice Grossberg, mutuamente constitutivos. 


\section{NOTAS}

${ }^{1}$ Las ideas fundamentales de este artículo fueron presentadas en el Congreso Internacional Transnacionalidad, diáspora y globalización en la cultura visual contemporánea, celebrado en la Universidad Autónoma de Madrid en octubre de 2009. La investigación fue llevada a cabo en el marco del proyecto de investigación del mismo nombre (CCG08-UAM/HUM-4426) financiado por la Comunidad de Madrid y dirigido por la profesora Patricia Mayayo. El presente artículo se ha escrito gracias a la financiación de una beca predoctoral del Programa de Formación del Profesorado Universitario (FPU) del Ministerio de Educación. Excepto que se especifique lo contrario, todas las traducciones son mías.

2 Chin-Tao Wu, "Worlds Apart. Problems of Interpreting Globalised Art", en Third Text 21(2), 2007, p. 719.

${ }^{3}$ Rasheed Araeen, "¿Cómo se ha convertido Third Text en Tercer Texto?", en Tercer Texto - Third Text: una perspectiva crítica sobre el arte y la cultura contemporáneos 1, 2003, p. 9.

${ }^{4}$ Jean Fisher, 'The Syncretic Turn: Cross-Cultural Practices in the Age of Multiculturalism", en Melina Kalinovska (ed.), New Histories, Londres, Institute of Contemporary Arts, 1996, p. 35, citado en Kobena Mercer, "Ethnicity and Internationality: New British Art and the Diaspora-based Blackness", en Rasheed Araeen, Sean Cubitt y Ziauddin Sardar (eds.), The Third Text Reader on Art, Culture and Theory, Londres y Nueva York, Continuum, 2005, p. 117.

${ }^{5}$ Tomo el concepto de "hipervisibilidad" de Kobena Mercer, Ibíd.

${ }^{6}$ Ian McLean, "On the Edge of Change? Art, Globalisation and Cultural Diffference", en Third Text 18 (3), 2004, p. 293.

7 Wu, "Worlds Apart", p. 721. Wu analiza los casos de Doris Salcedo, Shirin Neshat y Mona Hatoum.

${ }^{8}$ Ibid, p. 727.

${ }^{9}$ Sigo aquí el sentido que da a es- ta expresión Hans Belting, como una manera general de aludir a los cambios que ha sufrido la producción artística contemporánea debido a la globalización. Belting habla del "significado completamente distinto" que adquirió el arte contemporáneo cuando, "siguiendo el giro de la política y el comercio mundiales en 1989, se expandió a través del globo", y atribuye a este cambio fundamental el hecho de que ya no sea posible seguir adoptando una visión eurocéntrica del arte. Cfr. H. Belting "Contemporary Art as Global Art: A Critical Estimate", en H. Belting y A. Buddensieg (eds.), The Global Art World. Audiences, Markets, and Museums, Ostfildern, Hatje Cantz, 2009, p. 39.

${ }^{10}$ Hatoum consideró The Light at the End como "el principio de una manera completamente nueva de trabajar". Cf. "Michael Archer in conversation with Mona Hatoum", en Michael Archer, Guy Brett y Catherine de Zegher (eds.), Mona Hatoum, Londres, Phaidon, 2003, p. 17. Sobre la primera etapa de Hatoum centrada en vídeos y performances véase especialmente la primera parte de esa misma entrevista, pp. 8-14, así como Guy Brett, "A Hatoum Chronology", en Mona Hatoum, Bristol, Arnolfini, 1993. Para una contextualización de este tipo de obras audiovisuales y performativas: G. Brett, "Tissues of Thought. Performance and Some Other Works in London 1970-1985", Third Text, 22(2), 2008, pp. 237-256. Resulta llamativo el hecho de que mientras que las performances de Hatoum casi siempre se han analizado en relación a su contexto, no ha ocurrido lo mismo con las instalaciones.

${ }^{11}$ La obra se retransmitió en vivo desde Vancouver —donde la artista la realizaba como performance- a Viena. Para una análisis más detallado de esta obra donde se consideran otras posibles interpretaciones véase Jessica Morgan, "The Poetics of Uncovering", en Mona Hatoum, Chicago y Nueva York, Museum of Contemporary Art, 1997, p. 7.
12 "Con las instalaciones quería implicar al espectador en una situación fenomenológica en la que la experiencia es más física y directa. Quería que el aspecto visual de la obra comprometiera al espectador de un modo físico, sensual, quizá incluso emocional; las asociaciones y la búsqueda de significado vienen a continuación". En "Mona Hatoum entrevistada por Janine Antoni, Nueva York, primavera de 1998" en Mona Hatoum, Santiago de Compostela y Salamanca, Centro Galego de Arte Contemporánea y Centro de Arte de Salamanca, 2002, p. 122. Refiriéndose en concreto a The Light at the End, Hatoum ha subrayado que "no era tanto la representación de algo como la cosa en sí misma". "Michael Archer in conversation...", p. 17.

13 "En las primeras performances, me veía a mí misma como una persona marginal que intervenía desde dentro de los márgenes del mundo artístico, y parecía lógico utilizar las performance como una crítica al sistema. Pasado un tiempo, me iba sintiendo insatisfecha con la actitud obviamente retórica y ya no estaba segura de si la obra que estaba haciendo era realmente lo que quería hacer o el resultado de interiorizar las expectativas de otras personas y el hecho de que me habían encasillado en el papel del artista político". "[...] si procedes de un entorno acuciado por problemas se suele producir una expectativa en el sentido de que tu obra debería en cierto modo articular la lucha o representar la voz del pueblo". "Cuando mi obra pasó de una actitud obviamente política y retórica a una actitud que muestra las ideas políticas a través de lo formal y lo estético, la obra se convierte en un sistema más abierto. Desde entonces, me he resistido a los intentos de las instituciones de fijar el significado de mi obra al querer incluirla en exposiciones temáticas definidas con criterios muy estrechos. [...] Siempre he tenido una actitud contraria. Cuanto más siento que estoy siendo presionada para entrar en un molde, más deseo ir en la dirección opuesta". En "Mona Hatoum entrevistada 
por Janine Antoni", pp. 125, 117 y 123 respectivamente.

14 "La gente a menudo espera definiciones ordenadas de la otredad, como si la identidad fuera algo fijo y fácilmente definible". En "Mona Hatoum entrevistada por Janine Antoni", p. 117. Hatoum ha confesado haber tomado conciencia de esta tendencia a encasillarla en categorías como "artista política" cuando le ofrecieron participar en la exposición Nationalisms: Women and the State, que se celebró en Toronto en 1988: "Para mí ese fue realmente el principio del fin de trabajar de una manera abiertamente política". Hatoum en "Michael Archer in conversation...", p. 13.

${ }^{15} \mathrm{Wu}$, "Worlds Apart", p. 721

${ }^{16}$ Ibid.

${ }^{17}$ Mercer, "Ethnicity and Internationality", p. 116-118.

${ }^{18}$ Quizás el autor más importante en este sentido es Edward Said. Cf. E. Said, "The Art of Displacement: Mona Hatoum's Logic of Irreconcilables", en Stephen Deuchar (ed.), Mona Hatoum: The Entire World as a Foreign Land, Londres, Tate Gallery Publishing, 2000, pp. 7-17. Véase también en el mismo volumen el texto de Sheena Wagstaff, "Uncharted Territory: New Perspectives in the Art of Mona Hatoum", pp. 27-41.

${ }^{19}$ Wu, "Worlds Apart", p. 725.

20 Jessica Morgan, "The Poetics of Uncovering", p. 9.

${ }^{21}$ Esta cuestión ha sido puesta de manifiesto por Julian Stallabrass, High Art Lite: British Art in the 1990s. Londres y Nueva York, Verso, 2006 [1999], pp. 4-5. Stallabrass titula significativamente la primera parte de su libro con la expresión "saliendo de la recesión" [rising from recession]. Sobre el proceso de privatización que se inició con el gobierno de Margaret Thatcher Cf. Chin-Tao Wu, Privatising Culture: Corporate Art Intervention Since de 1980s, Londres, Verso, 2003.

${ }^{22}$ Rasheed Araeen, "A Very Special British Issue?: Modernity, Art History and the Crisis of Art Today", Third Text, 22(2), 2008, p. 138. La exposi- ción tuvo lugar en la Hayward Gallery de Londres entre noviembre de 1989 y febrero de 1990, luego rotó por otras galerías de Inglaterra. Véase The Other Story: Afro-Asian Artists in Post-war Britain, Londres, The Hayward Gallery, South Bank Centre, 1989. Para una visión crítica de la recepción de The Other Story, Cf. Rasheed Araeen, 'The Other Immigrant: The Experiences and Achievements of AfroAsian Artists in the Metropolis', Third Text, 5(15), 1991, pp. 7-28.

${ }^{23}$ Cf. Mark Crinson, " "Fragments of Collapsing Space": Postcolonial Theory and Contemporary Art", en Amelia Jones (ed.): A Companion to Contemporary Art Since 1945, Oxford, Blackwell, 2006, pp. 450-469.

${ }^{24}$ Uno de ellos es John Roberts, "Mad for It! Philistinism, the Everyday and the New British Art", Third Text, 35, 1996, pp. 29-42. También K. Mercer, "Ethnicity and Internationality", p. 120 , que sigue a Roberts en este punto, aunque no comparte su visión "neo-populista" del New British Art.

${ }^{25}$ Freedman, citado en Julian Stallabrass, High Art Lite, p. 55. Stallabrass menciona, incluso, la crítica que hicieron Carl Freeman y David Batchelor de la exposición Material Culture: The Object in British Art of the 1980s and 90s, en la que se presentaban obras tanto de la nueva generación de artistas británicos como de la anterior generación, a la que pertenecía propiamente Hatoum. En su artículo, Batchelor y Freedman ponen de manifiesto cómo a mediados de los años noventa - la exposición tuvo lugar en 1997- el arte político seguía sin despertar especial interés entre los críticos. Pese a su estética renovada, las obras de Hatoum seguían teniendo una clara alusión a la realidad política que ambos autores despreciaron. Refiriéndose a No Way y No Way III, dos piezas realizadas respectivamente con una espumadera y un colador cuyos agujeros han sido tapados por tornillos, Freedman y Batchelor escriben: "[la obra de Hatoum] representa la contención y las restricciones a las que se enfrentan las comunidades árabes en Jerusalén. Pero es estéril, forzada y cargada por su mensaje político [...]". D. Batchelor y C. Freedman, "Living in a Material World", Frieze, 35, 1997. Consultado en: http://www.frieze.com /issue/article/living_in_a_material_worl d/ (16.01.2010). Citado también en Stallabrass, de donde saco la referencia. High Art Lite, p. 3.

${ }^{26}$ Sobre la compleja relación del young British art con el minimalismo véase Hyla L. Robicsek, "'You Give Us This and We Give You That': 'young British art' ant the Legacy of AngloAmerican Influence", en Third Text, 22(2), 2008, pp. 257-271.

${ }^{27}$ Cf. "Postminimalism revisited. 1993b", en H. Foster, R. Krauss, Y-A. Bois y B.H.D. Buchloh, Art Since 1900. Modernism, Antimodernism, Postmodernism, Nueva York, Thames \& Hudson, 2004, pp. 635-638.

${ }^{28}$ Stallabrass, High Art Lite, p. 3.

${ }^{29}$ Tomo esta expresión de Anna C. Chave, "Minimalism and the Rhetoric of Power", Arts Magazine, 64(5)، 1990, pp. 44-63. Aunque no comparto enteramente el argumento de Chave, creo que es factible relacionar su visión crítica de minimalismo con esta obra de Hatoum, pues La Grande Broyeuse parece materializar los planteamientos de esta autora al hacer ostensible lo que de agresivo y opresor tiene el cuidado y aparentemente aséptico lenguaje formal del minimalismo. Sobre Party Time véase Robicsek, "You Give Us...", pp. 257 y 264-268, con bibliografía adicional, y los comentarios del propio Hirst en la entrevista que le hizo la revista The Idler el 10 de Julio de 1995: http://idler.co.uk/conversations /damien-hirst/

${ }^{30}$ Sobre las alusiones metafóricas de La Grande Broyeuse véase Laura Steward Heon "Grist for the Mill", en Mona Hatoum. Domestic Disturbance, North Adams, Mass, SITE Santa Fe, 2001, pp. 11-17. Véase también Tamar Garb, "Nostalgia de hogar", en Mona Hatoum, Santiago de Compostela y Salamanca, Centro Galego de Arte Contemporánea y Centro de Arte de Salamanca, 2002, pp. 17-31. 
${ }^{31}$ Art Since 1900, p. 637.

32 Lynn Zelevansky, Sense and Sensibility. Women Artists and Minimalism in the Nineties, Nueva York, The Museum of Modern Art, 1994, p. 8.

${ }^{33}$ Dave Hickey, The Invisible Dragon: Four Essays on Beauty, Los Angeles, Art issues. Press, 1993. Para una crítica de los planteamientos de Hickey desde el punto de vista de las políticas de identidad véase el texto de Amelia Jones " "Every man knows where and how beauty gives him pleasure": Beauty Discourse and the Logic of Aesthetics", publicado originalmente en $X$-Tra, 2(3). Se puede consultar una versión más reciente, revisada y corregida por la autora en: http:// www.strikingdistance.com/xtra/XTra100/v2n3/a jones3.html (23.07.2010).

${ }^{34}$ Hatoum en "Michael Archer in conversation...", p. 20.

${ }^{35}$ Ibid.

${ }^{36}$ Mercer, "Ethnicity and Internationality", p. 117.

${ }^{37}$ Homi Bhabha, El lugar de la cultura, Buenos Aires, Manantial, 2002, p. 112.

${ }^{38}$ Hyla Robicsek ha utilizado precisamente el concepto de mímica colonial de Bhabha para explicar la relación, la diferencia y la actitud del minimalismo británico de los noventa con respecto al minimalismo norteamericano, atribuyendo así a los young British artists el deseo de hacer "casi lo mismo, pero no exactamente", es decir, "usar el peso de la tradición" minimalista así como "la capacidad de re- conocimiento del original" con el fin de "socavarlo". Robicsek, "You Give Us...", p. 267, que recoge en este caso las palabras de Anthony Caro. Yo utilizo aquí el concepto de Bhabha de la misma forma, con esa misma capacidad subversiva, aunque luego le doy un sentido distinto al de Robicsek al plantear la posibilidad de que la posición subversiva de Hatoum se pierda en el proceso de recepción debido, precisamente, al hecho de ser "casi lo mismo".

${ }^{39}$ Interrogating Identity, Grey Art Gallery and Study Center, New York y Museum of Fine Arts, Boston, entre otros, 1991.

${ }^{40}$ Sense and Sensibility. Women Artists and Minimalism in the Nineties, Nueva York, The Museum of Modern Art, 1994. Resulta curioso que esta exposición haya sido relacionada con cierto internacionalismo porque se sumaría a otros dos fenómenos de internacionalismo diferentes de los que también participó Hatoum en los noventa: el de las bienales y el del nuevo arte británico. Sobre el internacionalismo de Sense and sensibility véase el comentario de Ayisha Abraham en S. Kolbowski, M. Nixon, M. Kelly, H. Foster, L. Kotz, S. Leung y A. Abraham, "A Conversation on Recent Feminist Art Practices", October, 71, 1995, p. 64.

${ }^{41}$ Forces of Change: Artists of the Arab World, The National Museum of Women in the Arts, Washington D.C., 1994.

${ }^{42}$ Norman Rosenthal, Sensation:
Young British Artists from the Saatchi Collection, Londres, Thames \& Hudson; Royal Academy of Arts, 1997.

${ }^{43}$ Wu, "Worlds Apart", pp. 727 y 731.

${ }^{44}$ El hecho de que las instalaciones de Hatoum tienen un sentido tan general que se vuelve prácticamente imposible entender de qué manera se relacionan con su origen o con cualquier cuestión política concreta ya fue subrayado por Silvia Kolbowski a mediados de los años noventa con motivo de la participación de Hatoum en la exposición Sense and Sensibility. Kolbowski entendía el cambio de la obra Hatoum desde las performances de los años ochenta a las instalaciones de los noventa como un movimiento desde la especificidad (asociada a cuestiones de diferencia sexual) a la generalidad (relacionada con un giro hacia la agresión). Cf. el comentario de Silvia Kolbowski en "A Conversation on Recent Feminist Art Practices", p. 63.

${ }^{45}$ Ian McLean, "On the Edge of Change?", p. 295.

${ }^{46}$ Gerardo Mosquera, "Notes on Globalization and Cultural Difference", en Nikos Papastergiadis, Complex Entanglements: Art, Globalisation and Cultural Difference, Londres, River Oram, 2003, citado en lan McLean, "On the Edge of Change?", p. 301.

47 Lawrence Grossberg, "Identity and Cultural Studies: Is That All There Is?", en Stuart Hall y Paul Du Gay (eds.), Questions of Cultural Identity, Londres, SAGE Publications, 1996, p. 90. 